\title{
Effectiveness of intraoperative ultrasonography for para-aortic lymph nodes in preventing unnecessary lymphadenectomy in ovarian carcinoma
}

\author{
Eiji Ryo ${ }^{1}$, Tsunekazu Kita ${ }^{1}$, Toshiharu Yasugi ${ }^{2}$, Katsumi Mizutani ${ }^{2}$, Michiharu Seto ${ }^{1}$, \\ Shigeki Takeshita ${ }^{1}$, Takuya Ayabe ${ }^{1}$ \\ ${ }^{1}$ Department of Obstetrics and Gynecology, Teikyo University, Tokyo, Japan \\ ${ }^{2}$ Department of Gynecology, Tokyo Metropolitan Cancer and Infectious Disease Center, Komagome Hospital, Tokyo, Japan \\ Email: yonch@med.teikyo-u.ac.jp
}

Received 18 April 2013; revised 20 May 2013; accepted 27 May 2013

Copyright (c) 2013 Eiji Ryo et al. This is an open access article distributed under the Creative Commons Attribution License, which permits unrestricted use, distribution, and reproduction in any medium, provided the original work is properly cited.

\begin{abstract}
Objective: To evaluate the usefulness of intraoperative ultrasonography (IU) for para-aortic nodes to identify women who do not require pelvic and paraaortic lymphadenectomy in ovarian carcinoma. Methods: Computed tomography (CT) was used for assessing both pelvic and para-aortic lymph nodes, and IU only for para-aortic nodes in 87 women with ovarian carcinoma. All women underwent surgery with routine systematic pelvic and para-aortic lymphadenectomy. We assumed that no lymphadenectomy had been performed when no enlarged node was detected by either CT or IU or when the woman was in T1 stage. Under these assumptions, the numbers of women who would have had missed metastases and who could have avoided lymphadenectomy were counted. These figures were recounted on the combination of $T$ stage and IU. Results: A total of 22 women had pathological node metastases. The numbers of women with missed metastases on the basis of CT, IU, and T stage were 12, 2, 5, and these who could have avoided lymphadenectomy were 72,39 , and 49 , respectively. There were more women avoiding lymphadenectomy by CT than IU and T stage; however, more women with missed node metastases. Both numbers were not significantly different between $\mathrm{IU}$ and $\mathrm{T}$ stage. On the combination of $T$ stage and IU, 29 of 49 women in T1 stage could have avoided lymphadenectomy without missed metastases. Conclusions: IU for the para-aortic node is a useful method for identifying women who do not require lymphadenectomy for $\mathrm{T} 1$ stage ovarian carcinoma.
\end{abstract}

Keywords: Intraoperative Ultrasonography;
Lymphadenectomy; Lymph Node; Lymph Node Metastases; Ovarian Carcinoma; Para-Aortic; Ultrasound

\section{INTRODUCTION}

In ovarian carcinoma, pelvic and para-aortic nodes are regional lymph nodes; whether or not they are metastasized is very important. However, to date, there is no definite protocol for assessing these lymph nodes.

Systematic lymphadenectomy is the most accurate method of assessment. However, routine lymphadenectomy has not become a standard procedure as it increases surgical morbidity and its therapeutic significance is unclear. A multivariable analysis study [1] reported that the extent of lymphadenectomy was a significant prognostic factor for improved survival in clinical stage I ovarian cancer. Similarly, patients with ovarian carcinoma upgraded to stage III following systematic lymphadenectomy had similar superior survival to stage I and II patients [2]. On the other hand, a randomized study of systematic lymphadenectomy was conducted in patients with epithelial ovarian cancer macroscopically confined to the pelvis, and it did not exhibit any effects on survival [3]. Panici et al. carried out a prospective randomized study of women with optimally operated advanced ovarian carcinoma and reported that when compared to resection of bulky nodes only, systematic lymphadenectomy improved progression-free but not overall survival [4].

It is generally accepted that common imaging modalities such as CT, ultrasonography, MRI, and scintigraphy have low sensitivity for detecting node metastases in gynecological malignancies [5,6]. A recent study reported that PET/CT has high sensitivity (84\%) for lymph node metastases in ovarian carcinoma; however, routine systematic lymphadenectomy was not used as the reference 
standard [7].

Like other malignancies, the incidence of node involvement increases as the primary intraabdoninal disease, which means $\mathrm{T}$ stage, progresses in ovarian carcinoma. However, there is no protocol for identifying women who do not need lymphadenectomy on the basis of T stage.

In our previous studies, we examined the ability of intraoperative ultrasonography (IU) to detect the enlarged para-aortic lymph node in women with gynecological malignancies [8]. IU had superior negative predictive value and sensitivity for para-aortic node metastases. In addition, IU was the most useful diagnostic tool for avoiding unnecessary para-aortic lymphadenectomy in women with endometrial carcinoma [9].

In ovarian carcinoma, the para-aortic node is the most frequent site of lymph node metastases. The purpose of this study was to investigate the usefulness of IU for avoiding unnecessary lymphadenectomy in women with ovarian carcinoma, as well as to compare its use with that of CT and T stage.

\section{MATERIALS AND METHODS}

At our hospital, all women below 75 years of age who have ovarian carcinoma and good performance status undergo systematic pelvic and para-aortic lymphadenectomy up to the renal vessels, when abdominal residual tumors are less than $1 \mathrm{~cm}$ in diameter and no distant etastases are present. Under these criteria, 87 women underwent lymphadenectomy at Teikyo University Hospital between 2002 and 2010. The mean age (standard deviation) of the women was 54.2 (10.60) years. Table 1 lists their clinical characteristics. All women provided informed consent, and the institutional review board approved the study.

CT was performed in the pelvic and para-aortic region with a helical a multidetector-row 8-channel helical CT unit (Light Speed QX/I; GE Medical Systems, Milwaukee, WI, USA) within the 2-week period before surgery. All women received $2 \mathrm{~mL} / \mathrm{kg}$ non-ionic intravenously administrated contrast material. Scanning was performed between 90 and 120 seconds after the initiation of intravenous contrast administration. The assessments whether the pelvic and para-aortic lymph nodes were enlarged were performed by radiologists with more than 10 years of experience.

IU for para-aortic nodes was performed as described previously [8,9]. A patient was placed in the lithotomy position under general anesthesia. After the lower abdomen was opened via a midline incision, an examiner inserted an intraoperative ultrasound probe into the upper abdomen. Following contact with the retroperitoneum, the examiner observed the aortic bifurcation, inferior
Table 1. Characteristics of study population.

\begin{tabular}{cc}
\hline Variable & Number of patients (n) \\
\hline Total & 87 \\
FIGO stage & 42 \\
I & 6 \\
a & 2 \\
b & 34 \\
c & 8 \\
II & 0 \\
a & 1 \\
b & 7 \\
c & 34 \\
III & 0 \\
a & 5 \\
b & 29 \\
c & 3 \\
IV & \\
Pathology & 27 \\
Serous & 30 \\
clear & 7 \\
mucinous & 10 \\
Endometrium & 7 \\
Poorly differentiated & 6 \\
Others &
\end{tabular}

mesenteric artery, renal arteries and veins, and bilateral ovarian veins that could be observed via IU, and confirmed the para-aortic area. Then, the area was scanned by moving the ultrasound probe on the transverse plane. If a well-defined, hypoechoic, apparently round structure was found, its shape was confirmed to be round by further observations from more than 2 different directions to exclude vessels. In some cases, color Doppler imaging was used to distinguish between lymph nodes and vessels by observing the blood flow. A well-defined, hypoechoic round structure adjacent to the aorta or the vena cava was identified as a lymph node and was assessed as enlarged if its diameter in the transverse plane exceeded $5 \mathrm{~mm}$. All scanning procedures were performed by a gynecological doctor, and a NEMIO SSA 550A system with a PVF-738H probe (Toshiba Medical System, Tokyo, Japan) was used.

Subsequently IU, intra-pelvic procedures such as hysterectomy, bilateral salpingo-oophorectomy, and pelvic lymphadenectomy were carried out. Then, the abdominal incision was extended to the subxiphoid region, and omentectomy and systematic para-aortic lymphadenectomy were performed in all women. All removed tissues including lymph nodes were examined microscopically, and the numbers of women with node metastases were counted related to $\mathrm{T}$ stage and their sites. From intraabdominal findings, we classified the women into a lowrisk group for node metastases when the surgical staging was T1.

First, the diagnostic accuracies assessed by measures such as sensitivity, specificity, and positive and negative predictive values of $\mathrm{CT}$, IU, and T stage for node metastases were calculated. 
Next, we assumed that no lymphadenectomy had been performed when no enlarged pelvic and para-aortic lymph node was detected by CT. In the same way, we assumed no lymphadenectomy when IU detected no enlarged para-aortic node or when the women were classified into T1 stage. We calculated the numbers of women who would have had missed node metastases and who could have avoided lymphadenectomy, and compared them using the chi-squared and Fisher's exact probability test. Statistical significance was set at $\mathrm{p}<0.05$. Furthermore, we recounted these figures on the basis of the combination of $\mathrm{T}$ stage and IU.

\section{RESULTS}

There were lymph node metastases in 22 (25.3\%) of 87 women. The mean (standard deviation) numbers of pelvic and para-aortic nodes removed per patient were 36 (11.3) and 24 (12.7), respectively. Table 2 states the number of women with node metastases related to the $\mathrm{T}$ stage and their sites. The rates of lymph node metastases were $10.2 \%, 22.2 \%$, and $51.7 \%$ for the $\mathrm{T} 1, \mathrm{~T} 2$, and $\mathrm{T} 3$ stages, respectively. Para-aortic node metastases only were found in 12 women, and both pelvic and para-aortic metastases were present in 9. Thus, 21 patients in total had para-aortic node metastases. A single case had pelvic node metastases only.

IU took less than five minutes and there was no adverse effect in all women. Figure 1 shows an example of the enlarged para-aortic lymph node detected by IU.

Table 3 shows the sensitivity, specificity, and positive and negative predictive values of CT, IU, and T stage for node metastases. No single method was superior with respect to all diagnostic indices. Both sensitivity and negative predictive value of IU were more than $90 \%$.

No enlarged lymph node was detected by CT in 72 women, and 12 of these had metastasized nodes. Theoretically, this means that if lymphadenectomy had been performed only when enlarged lymph nodes were detected by CT, 72 women (82.8\%) could have avoided lymphadenectomy. However, node metastases would have been missed in 12 out of 22 women with metastases (Table 4).

In the same way, the numbers of women with missed

Table 2. Number of women with node metastases related to $\mathrm{T}$ stage and their sites.

\begin{tabular}{cccccc}
\hline $\begin{array}{c}\mathrm{T} \\
\text { stage }\end{array}$ & Number & PAL only & PLN only & PAL \& PLN & Total \\
\hline $\mathrm{T} 1$ & 49 & 4 & 0 & 1 & $5(10.2 \%)$ \\
$\mathrm{T} 2$ & 9 & 1 & 0 & 1 & $2(22.2 \%)$ \\
$\mathrm{T} 3$ & 29 & 7 & 1 & 7 & $15(51.7 \%)$ \\
Total & 87 & 12 & 1 & 9 & 22 \\
\hline
\end{tabular}

PAL = Para-aortic node; PLN = Pelvic node.

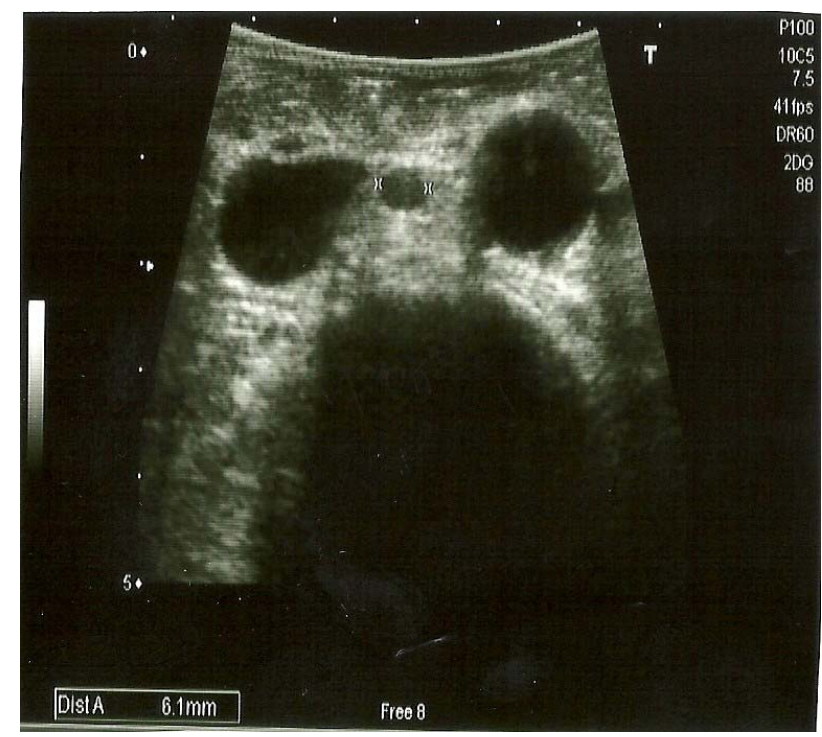

Figure 1. Transverse section of the para-aortic lymph node. It is $6.1 \mathrm{~mm}$ in diameter and is located between the vena cava and aorta.

Table 3. Diagnostic accuracy of CT, IU, and T stage for node metastases.

\begin{tabular}{ccccc}
\hline \multirow{2}{*}{ Method } & $\begin{array}{c}\text { Sensitivity } \\
\%(\mathrm{n})\end{array}$ & $\begin{array}{c}\text { Specificity } \\
\%(\mathrm{n})\end{array}$ & $\begin{array}{c}\text { PPV } \\
\%(\mathrm{n})\end{array}$ & $\begin{array}{c}\text { NPV } \\
\%(\mathrm{n})\end{array}$ \\
\hline \multirow{2}{*}{$\mathrm{CT}$} & 45.5 & 92.3 & 66.7 & 83.3 \\
& $(10 / 22)$ & $(60 / 65)$ & $(10 / 15)$ & $(60 / 72)$ \\
IU & 91.0 & 56.9 & 41.7 & 94.9 \\
& $(20 / 22)$ & $(37 / 65)$ & $(20 / 48)$ & $(37 / 39)$ \\
\multirow{2}{*}{ T stage } & 77.3 & 67.7 & 44.7 & 89.8 \\
& $(17 / 22)$ & $(44 / 65)$ & $(17 / 38)$ & $(44 / 49)$ \\
\hline
\end{tabular}

$\mathrm{PPV}=$ positive predictive value, $\mathrm{NPV}=$ negative predictive value.

Table 4. Numbers of women who could have avoided lymphadenectomy and would have had missed metastases on the basis of CT, IU, and T stage.

\begin{tabular}{ccc}
\hline Method & $\begin{array}{c}\text { Lymphadenectomy } \\
\text { avoided (\% per total) }\end{array}$ & $\begin{array}{c}\text { Missed metastases } \\
\text { (\% per positive metastases) }\end{array}$ \\
\hline CT & $72(82.8)$ & $12(54.5)$ \\
IU & $39(44.8)$ & $2(9.0))$ \\
T stage & $49(56.3)$ & $5(22.7)$ \\
\hline P value & & 0.004 \\
\hline CT-IU & $<0.001$ & 0.409 \\
IU-T stage & 0.129 & 0.03 \\
T stage-CT & $<0.001$ & \\
\hline
\end{tabular}

A total of 87 women actually underwent lymphadenectomy and metastases were found in 22; CT = Computed Tomography, IU = Intraoperative Ultrasonography.

metastases on the basis of IU and T stage were 2 and 5 , and the numbers of women avoiding lymphadenectomy were 39 and 49, respectively (Table 4).

There were more women who could have avoided lymphadenectomy by CT than IU and T stage; however, more women with missed node metastases. Both numbers were not significantly different between IU and $\mathrm{T}$ 
stage.

As for 2 women with missed metastases on the basis of IU, one had solely pelvic node metastases and the other had para-aortic metastases.

On the combination of T stage and IU, 29 (59.2\%) of 49 women in $\mathrm{T} 1$ stage could have avoided lymphadenectomy without missed metastasis by IU. For the T2-3 stages, 10 (26.3\%) of 38 women could have avoided lymphadenectomy, and 2 would have had missed metastases (Table 5).

\section{DISCUSSION}

No single method was superior with respect to all diagnostic indices. Thus, a postoperative staging system without lymphadenectomy is unreliable; however, it increases surgical morbidities and is not therapeutically significant for all patients. Lymphadenectomy may have a prognostic benefit for women with node metastases but has no value for women without them. In this study, 65 (74.7\%) of 87 women with no node metastases were subjected to unnecessary surgical morbidities as a result of routine lymphadenectomy. It would be useful to be able to identify women who do not require this procedure.

For procedures in which lymphadenectomy is performed only in women with suspected node metastases indicated by any method, the positivity of metastases is clarified by microscopic examinations of removed lymph nodes. Thus, specificity turns out to be perfect by eliminating false positives. On the contrary, negativity of metastases cannot be confirmed because lymphadenectomy is omitted. It means sensitivity and negative predictive value are more important than the other indices. Both sensitivity and negative predictive value were more than $90 \%$ in IU.

There were more women with missed metastases on the basis of CT compared with IU and T stage. Sensitivity of CT was less than 50\%. We thought that it was not appropriate to depend on CT to identify women who do not need lymphadenectomy.

As with other malignancies, the incidence of node involvement in ovarian carcinoma increases as the $\mathrm{T}$ stage progresses. According to the total of several previous studies [10-15], the risk of node metastasis in 722 women was $13.0 \%, 37.3 \%$, and $64.0 \%$ in $\mathrm{T} 1$, T2, and $\mathrm{T} 3$, respectively. Intra-abdominal primary tumor staging is

Table 5. Number of women who could have avoided lymphadenectomy and would have had missed node metastases by intraoperative ultrasonography related to T stage.

\begin{tabular}{cccc}
\hline & Number & Lymphadenectomy avoided & Missed metastases \\
\hline T1 & 49 & $29(59.2 \%)$ & 0 \\
T2-3 & 38 & $10(26.3 \%)$ & 2 \\
Total & 87 & $39(44.8 \%)$ & 2 \\
\hline
\end{tabular}

an important predictor for lymph node metastases; however, the metastatic rate is greater than $10 \%$ even in the T1 stage, and the results of our study were consistent with these findings. From viewpoint of therapy, it is reported that women who upstaged to stage III only after systematic lymphadenectomy have similar superior survival like stage I and II patients [2]. This indicates the prognostic effect of systematic lymphadenectomy in T1N1 patients. Similarly, women with missed node metastases on the basis of $\mathrm{T}$ stage might lose a chance to receive postoperative chemotherapy; this is because with the same findings, they would have been considered to have a low risk for recurrence. $\mathrm{T}$ stage is a fair predictor for lymph node metastases, but there are weak points in determining women who do not undertake lymphadenectomy only by that.

Tangitgamol et al. [16] reported that, in histological sections, more than half of the positive nodes have a nodal long axis of $10 \mathrm{~mm}$ or less, and concluded that node size is an unreliable indicator of metastases in ovarian carcinoma. Indeed, lymph nodes less than $10 \mathrm{~mm}$ are too small to estimate by common imaging modalities such as CT, MRI, and transabdominal ultrasonography. In transabdominal ultrasonography, the abdominal wall and bowels attenuate and scatter ultrasound, which produces low-quality images. However, this can be avoided by bringing the ultrasound probe close to the target in an intraoperative approach such as transvaginal ultrasonography. In obstetric practice, transvaginal ultrasonography with high-frequency ultrasound measures fetal nuchal translucency at intervals of $0.1 \mathrm{~mm}$, not $1 \mathrm{~mm}$. In cervical lymph node assessments in patients with head and neck cancers, the ultrasound probe can be close to the target, and ultrasonography has the highest sensitivity for metastases compared with CT, MRI, and PET/CT [17].

As Figure 1 illustrates, IU has the ability to estimate lymph nodes less than $10 \mathrm{~mm}$. In this study, there were histological para-aortic lymph node metastases in 21 women, and IU highlighted enlarged nodes in 20 of them. This demonstrates that IU has superior sensitivity for para-aortic lymph node metastases in ovarian carcinoma.

Scrutiny of node metastasis sites has revealed that para-aortic nodes are the most frequent sites of lymphatic metastases. In the above mentioned studies [10-15], the total risk of node metastases to solely para-aortic node, both pelvic and para-aortic, and solely pelvic were $11.4 \%$, $23.1 \%$, and $5.0 \%$, respectively. The number of women who had pelvic node metastases only was relatively small in ovarian carcinoma. Only one woman had solely pelvic node metastases in our study.

IU has superior sensitivity for the para-aortic node metastases, and a small number of women had solely pelvic node metastases. It follows that IU for the para- 
aortic regions may be useful to avoid unnecessary pelvic and para-aortic lymphadenectomy. In our results, if lymphadenectomy had been performed only when IU detected enlarged para-aortic lymph nodes, nearly half of the women could have avoided lymphadenectomy, and high sensitivity for detecting node metastases would still have been maintained. IU has proven to be a good method, though there were 2 cases that would have missed metastases in our study, and one of these had pelvic node metastases only.

Indeed the number of women with exclusively pelvic node metastases was relatively small; however, this number would increase as the primary intra-abdominal disease progresses. According to the above mentioned studies in total [10-15], the risks of solely pelvic node metastases were $2.9 \%(9 / 308), 6.7 \%(5 / 75)$ and 6.5\% (22/ 339) for T1, T2, and T3, respectively. It is evident from these data that if lymphadenectomy was performed based only on the result of IU scanning for the para-aortic node, the risk of missed pelvic node metastases would exceed $5 \%$ for the $\mathrm{T} 2$ and 3 stages. IU for the para-aortic region was a fair predictor for retroperitoneal node metastases like $\mathrm{T}$ stage, but we thought we cannot depend only on that.

Then, how about the combination of $\mathrm{T}$ stage and IU? The main lymphatic flow route from the ovary goes along with the ovarian vessels, and to the para-aortic regions. It is assumed that most pelvic node metastases occur only after the para-aortic nodes have been metastasized or the primary disease spreads to pelvic organs such as the peritoneum and uterus. The para-aortic nodes should be sentinel ones for $\mathrm{T} 1$ stage of ovarian carcinoma. This means that lymphadenectomy may be omitted in cases without para-aortic node enlargement especially for the T1 stage. In our results, no woman in the T1 stage had solely pelvic node metastases, and more than half of the women in this stage could have avoided lymphadenectomy without missed metastases by IU. We found IU was a useful method for identifying women who do not require lymphadenectomy in T1 stage ovarian carcinoma.

IU has the best sensitivity for para-aortic node metastases in gynecological malignancies [8]. Ultrasound machine is inexpensive and popular in world-wide. IU for the para-aortic area is a safe and easy procedure to master following basic instruction, because this area is flat, the branching pattern of the vessels is simple, and the image of enlarged lymph nodes are also simple. On the contrary, in our experience, IU for pelvic nodes was doable but not easily mastered procedures because the pelvic cavity is curved and the vessel patterns are complicated.

In conclusion, IU for the para-aortic node is a useful method for identifying women who do not need pelvic and para-aortic lymphadenectomy especially for T1 stage in ovarian carcinoma.

The authors declare that there were no conflicts of interest.

\section{REFERENCES}

[1] Chan, J.K., Munro, E.G., Cheung, M.K., Husain, A., Teng, N.N., Berek, J.S., et al. (2007) Association of lymphadenectomy and survival in stage I ovarian cancer patients. Obstetrics \& Gynecology, 109, 9-12.

[2] Onda, T., Yoshikawa, H., Yasugi, T., Mishima, M., Nakagawa, S., Yamada, M., et al. (1998) Patients with ovarian carcinoma upstaged to stage III after systematic lymphadenectomy have similar survival to stage I/II patients and superior survival to other stage III patients. Cancer, 83, 1555-1560.

doi:10.1002/(SICI)1097-0142(19981015)83:8<1555::AI D-CNCR10>3.0.CO;2-R

[3] Maggioni, A., Benedetti Panici, P., Dell’Anna, T., Landoni, F., Lissoni, A., Pellegrino, A., et al. (2006) Randomized study of systematic lymphadenectomy in patients with epithelial ovarian cancer macroscopically confined to the pelvis. British Journal of Cancer, 95, 699704. doi:10.1038/sj.bjc.6603323

[4] Panici, P.B., Maggioni, A., Hacker, N., Landoni, F., Ackermann, S., Campagnutta, E., et al. (2005) Systematic aortic and pelvic lymphadenectomy versus resection of bulky nodes only in optimal debulked advanced ovarian cancer: A randomized clinical trial. Journal of the $\mathrm{Na}$ tional Cancer Institute, 97, 560-566.

doi:10.1093/jnci/dji102

[5] Tempany, C.M.C., Zou, K.H., Silverman, S.G., Brown, D.L., Kurts, A.B. and Mcneil, B.J. (2000) Staging of advanced ovarian cancer: Comparison of imaging modalities; report from the Radiological Diagnostic Oncology Group. Radiology, 215, 761-767.

[6] Ozalp, S., Yalcin, O.T., Polay, S., Aslan, N., Vardareli, E. and Adpinar, B. (1999) Diagnostic efficacy of the preoperative lymphoscintigraphy, Ga-67 scintigraphy and computed tomography for detection of lymph node metastasis in cases with ovarian or endometrial carcinoma. Acta $\mathrm{Ob}$ stetricia et Gynecologica Scandinavica, 78, 155-159. doi:10.1080/j.1600-0412.1999.780215.x

[7] Nam, E.J., Yun, M.J., Oh, Y.T., Kim, J.W., Kim, J.H., Kim, S., et al. (2010) Diagnosis and staging of primary ovarian cancer: Correlation between PET/CT, Doppler, and CT or MRI. Gynecologic Oncology, 116, 389-394. doi:10.1016/j.ygyno.2009.10.059

[8] Ryo, E. (2008) Diagnostic value of intraoperative ultrasonography to assess para-aortic lymph nodes in women with ovarian and uterine corpus malignancy. Ultrasound in Obstetrics \& Gynecology, 32, 91-96. doi:10.1002/uog.5366

[9] Ryo, E., Yasugi, T., Mizutani, K., Kita, T., Takeshita, S. and Ayabe, T. (2011) Diagnostic usefulness of intraoperative ultrasonography in avoiding unnecessary paraaortic lymphadenectomy in women with endometrial carcinoma. International Journal of Gynecological Cancer, 


\section{1, 859-863. doi:10.1097/IGC.0b013e31821a35ef}

[10] Onda, T., Yoshikawa, H., Yokota, H., Yasugi, T. and Taketani (1996) Assessment of metastases to aortic and pelvic lymph nodes in epithelial ovarian carcinoma. A proposal for essential sites for lymph node biopsy. Cancer, 78, 803-808.

doi:10.1002/(SICI)1097-0142(19960815)78:4<803::AIDCNCR17>3.0.CO;2-Z

[11] Suzuki, M., Ohwada, M., Yamada, Kohno, T., Sekiguchi, I. and Sato, I. (2000) Lymph node metastasis in stage I epithelial ovarian cancer. Gynecologic Oncology, 79, 305308. doi:10.1006/gyno.2000.5951

[12] Burghardt, E., Girardi, F., Lahousen, M., Tamussino, K. and Stettner, H. (1991) Patterns of pelvic and paraaortic lymph node involvement in ovarian cancer. Gynecologic Oncology, 40, 103-106. doi:10.1016/0090-8258(91)90099-Q

[13] Morice, P., Joulie, F., Camatte, S., Atallah, D., Rouzier, R. and Pautier, P. (2003) Lymph node involvement in epithelial ovarian cancer: Analysis of 276 pelvic and paraaortic lymphadenectomies and surgical implications. Journal of the American College of Surgeons, 197, 198-205. doi:10.1016/S1072-7515(03)00234-5
[14] Negishi, H., Takeda, M., Fujimoto, T., Todo, Y., Ebina, Y., Watari, H., et al. (2004) Lymphatic mapping and sentinel node identification as related to the primary sites of lymph node metastasis in early stage ovarian cancer. $G y$ necologic Oncology, 94, 161-166. doi:10.1016/j.ygyno.2004.04.023

[15] Tsumura, N., Sakuragi, N., Hareyama, H., Satoh, C., Oikawa, M. and Yamada, H. (1998) Distribution pattern and risk factors of pelvic and para-aortic lymph node metastasis in epithelial ovarian carcinoma. International Journal of Cancer, 79, 526-530.

doi:10.1002/(SICI)1097-0215(19981023)79:5<526::AIDIJC14>3.0.CO;2-\#

[16] Tangitgamol, S., Manusirivithaya, S., Sheanakul, C., Leelahakorn, S., Sripramote, M., Thawaramata, T., et al. (2003) Can we rely on the size of the lymph node in determining nodal metastasis in ovarian carcinoma? International Journal of Gynecological Cancer, 13, 297-302. doi:10.1046/j.1525-1438.2003.13192.x

[17] Ahuja, A.T., Ying, M., Ho, S.Y., Antonio, G., Lee, Y.P., King, A.D., et al. (2008) Ultrasound of malignant cervical lymph nodes. Cancer Imaging, 8, 48-56. doi:10.1102/1470-7330.2008.0006 